\title{
Impact of Pharmacist Delivered Education on Early Parent Awareness and Perceptions Regarding Human Papillomavirus (HPV) Vaccination in the Community Pharmacy Setting in West Virginia
}

Jennifer A Wick, PharmD, MPH; Betsy M Elswick, PharmD

West Virginia University School of Pharmacy, Department of Clinical Pharmacy, Morgantown, WV

\begin{abstract}
Objectives: (1) Define parental perceptions of the Human Papillomavirus Vaccine and awareness of vaccine administration at community pharmacies (2) Describe parental intentions to have children vaccinated against HPV (3) Describe reasoning process behind parental vaccination intentions (4) Assess impact of pharmacist-led education on these perceptions and intentions

Methods: This was a prospective pretest, posttest study with a convenience sample conducted at parenting groups throughout northern West Virginia in 2018. Participants, a total of 34 parents/guardians, attended an educational session regarding Human Papillomavirus (HPV) vaccination with immediate pre/post survey. The survey asked participants about their HPV vaccination history, personal perceptions regarding the HPV vaccine, age and gender of their children, overall immunization status of the child, current intent regarding the HPV vaccine, parents' preferred resources for vaccine information, awareness of HPV vaccine availability in community pharmacies, as well as parent developed environment (rural, suburban, urban, etc.), race, age, marital status, education, and income level.
\end{abstract}

Results: Following intervention, intention to vaccinate according to the Advisory Committee on Immunization Practices (ACIP) recommendations increased from 35\% $(n=12)$ to $44 \%(n=15)$. The percentage of participants against vaccinating decreased from $23 \%$ $(n=8)$ to $12 \%(n=4)$. Participants demonstrated increased awareness of HPV vaccine availability at community pharmacies, with awareness increasing from $32 \%(n=11)$ to $100 \%(n=34)$.

Conclusions: Pharmacist delivered education may be useful in increasing parent/legal guardian awareness of immunization services as well as intention to vaccinate their child.

Key Words: Human Papillomavirus, Vaccination, Pharmacist, Community Education

\section{Introduction}

Vaccination against communicable diseases and the subsequent decrease in mortality and morbidity from these diseases has often been viewed as the magnum opus of public health. Public health practitioners have not only been able to see decreases in negative health outcomes from communicable diseases, but also the eradication of disease itself (as exemplified by smallpox $)^{1}$. However, achieving goal vaccination rates for various diseases remains a struggle. Vaccination of the public against the human papillomavirus (HPV) is one such disease that remains suboptimal.

Despite data supporting the use of the vaccine to prevent various types of cancer and genital warts ${ }^{2}$, vaccination rates remain far below Healthy People 2020 goals. Healthy People goals are proposed by a consortium of health experts including the Centers for Disease Control and Prevention (CDC), Food and Drug Administration (FDA), and National Institute of Health (NIH) among others. The goals are established each decade as

Corresponding author: Jennifer A Wick, PharmD, MPH Palm Beach Atlantic University, Department of Pharmacy Practice, West Palm Beach, FL; Email: jennifer wick@pba.edu a benchmark for achieving a healthier population in a variety of topics. The goal for complete HPV vaccination coverage (3 shot series according to available CDC vaccine schedule at time of goal authorship) is $80 \%^{3}$. In West Virginia, just over $60 \%$ of female adolescents and $45 \%$ of males aged $13-17$ have started the HPV series according to the 2015 National Immunization Survey-Teen (NIS-Teen) ${ }^{4}$. However, $74 \%$ of females and $69 \%$ of males completed the HPV three-dose series after initiation, showing that completion rates are promising for those who begin vaccination. West Virginia is also a primarily rural state, plagued by a lack of education regarding HPV. In a recent analysis of Health Information National Trends Survey (HINTS) data, only $55.8 \%$ and $58.6 \%$ of rural residents respectively were even aware of HPV and the existence of an HPV vaccine ${ }^{5}$. Meanwhile, West Virginia remains among the top states regarding HPV related oropharyngeal cancer, HPV related cervical cancer, and cervical cancer deaths according to the CDC (rates of 5.1, 9.9, and 2.4 per 100,000 respectively) ${ }^{6,7}$. Pharmacists can play a key role in providing patient interaction and education regarding the HPV vaccine.

Previous studies by Islam, McLean, and Gilkey have shown that convenient vaccination in pharmacies and strong professional recommendations can greatly increase vaccine uptake ${ }^{8-10}$. Yet, uptake remains low despite increases in access and the 
availability of studies supporting vaccination by pharmacists. A qualitative study by Westrick in Alabama has shown that misinformation and lack of trust in pharmacists' clinical qualifications are major barriers to vaccine uptake in the community pharmacy setting ${ }^{11}$. Education is needed to combat misinformation regarding vaccines and pharmacists' clinical abilities. Studies by Winer, Reiter, and Kester have shown that group educational efforts can increase HPV vaccination knowledge as well as intention to vaccinate ${ }^{12-14}$. In addition, studies by Loudon, Glanz, and Brunson have indicated that vaccination research and decisions are often made before the child is born or in early childhood ${ }^{15-17}$. Such information can be used to tailor immunization-based educational interventions for maximal impact by incorporating appropriate timing into intervention design.

While each of these studies addressed components of HPV vaccine education and intervention, none attempted such interventions with parents at such an early stage. Considering the aforementioned study outcomes, it was imperative to educate parents during very early childhood about the HPV vaccine. Furthermore, the HPV vaccine could not be delivered in the community pharmacy setting in West Virginia until 2018. Thus, no research or education about such access improvements had been completed. This study sought to demonstrate the impact of a pharmacist-led group educational intervention on HPV vaccine awareness and intent of parents/legal guardians of young children (those under the age of 11-12).

\section{Methods}

This was a prospective pretest, posttest study with a convenience sample conducted at parenting groups throughout northern West Virginia. The research protocol was approved by the Institutional Review Board of West Virginia University. Parenting groups contacted were formed for a variety of reasons. Common reasons cited for group formation were common interests (i.e. hiking), socializing the young children (play groups), or involvement in a community organization (church groups or groups involved with high school child development classes). A preliminary list of parenting groups was compiled using an internet based search engine. Groups were recruited in a multimodal approach; utilizing phone, email, and social media. Parents were informed of the educational session date by group administrators/facilitators. All children in these respective groups were under the age of 9 , the youngest age for which the HPV vaccine is approved. Educational sessions were conducted during normal school hours; thus, the majority of children present at each group were aged 0-6. Of note, some children did have siblings within the target age range for HPV vaccination, however families were not stratified based upon this data. This project utilized primarily quantitative methods, with the option for additional open responses on several items. When examining the reasoning behind decisions to avoid vaccination, open response items are imperative. It is vital to analyze not only the percentage of participants who choose not to vaccinate, but to determine why they are not vaccinating. Such data could be used to shape future research in the field.

This study targeted parents with young children, who would be eligible for the HPV vaccine in the future. Parents were excluded for inability (cannot write/read English) or unwillingness to complete the measurement tool, i.e. written survey. They were also excluded due to previous participation if the parent was present in multiple parent groups selected for intervention. Goal enrollment for the program was approximately 20 participants. This was based upon the time and space requirements for program conduction, as well as response rates to residency-based research projects in years prior. This goal was later surpassed as 34 parents participated. All parents who completed the educational intervention completed the survey. One parent left prior to the educational session beginning after being alerted by parent group administrators of an injury to her child during the playgroup (conducted by parent group administrators/facilitators simultaneously to provide child-care). This parent did not return to complete either the survey or the educational session.

Data collection was performed via written survey. The survey was a 17-item tool, which covered various items assessing vaccination history, perceptions, and intent as well as demographic information. The survey asked participants about their HPV vaccination history, personal perceptions regarding the HPV vaccine, age and gender of their children, overall immunization status of the child, current intent regarding the HPV vaccine, parents preferred resources for vaccine information, awareness of HPV vaccine availability in community pharmacies, as well as parent developed environment (rural, suburban, urban, etc.), race, age, marital status, education, and income level. Items were multiple choice, select all that apply, and open response. Questions were developed independently by the study team and informed by HINTS and previous qualitative interviews by Brunson et al. ${ }^{15}$ The survey was assessed for both face and content validity. Content was reviewed by an expert panel of frontline immunizers active in clinical practice, i.e. involved in state immunization organizations/efforts or clinical management roles. The survey was administered to participants prior to the educational session and immediately following the session. The program lasted approximately 30 minutes and included a short educational presentation (Microsoft PowerPoint ${ }^{\circ}$ ) with additional time allotted for questions to the pharmacist. The program addressed HPV and related cancer prevalence in West Virginia, cervical cancer mortality in West Virginia, available HPV vaccine products, vaccine dosing schedule, locations for vaccination, vaccine efficacy and safety, and sexual behaviors in the context of HPV vaccination. The educational program was 
pharmacist-taught and delivered to multiple parent groups in West Virginia. The pharmacist responsible for educational sessions was a community-based pharmacy resident with a Master's in Public Health focusing on health behaviors. Educational programming and survey distribution took place from January to May 2018 with 4 total sessions occurring. Final data analysis was performed in May 2018. Due to the use of categorical data, convenience sampling methods, small sample size, and lack of a control group, descriptive statistics alone were used. Percentages for each response were calculated and pre/posttest statistics compared using Microsoft Excel ${ }^{\circ}$.

\section{Results}

\section{Participation}

Out of nineteen parenting groups contacted for participation in the study, five (26\%) declined to participate. Each of these groups cited religious and political beliefs as reasons for declining. Eleven groups (58\%) did not respond to the invitation to participate for unknown reasons. Only three groups (16\%) opted to participate, with one group hosting at three separate locations and the remaining two groups hosting at one site each. Of the participating groups, one group did not achieve any participants, likely due to a scheduling issue. The organization had also scheduled an off-site field trip for all the children during the educational session.

\section{Demographics}

Of the participating parents/legal guardians, $91 \%(n=31)$ were Caucasian. This is in line with the locale's primarily Caucasian population. The remaining participants were of Native American or Asian/Pacific Islander descent $(n=3)$. The study population was also relatively homogenous in age. Forty-one percent $(n=14)$ of the population was between the ages of 2534 and an additional $32 \%(n=11)$ was between the ages of 35 44. This result was expected considering the usual age at childbirth and our target population (parents with children under the age of 11-12). The study population was relatively diverse in their educational levels. Fifteen percent $(n=5)$ of the population had attained a graduate/professional degree, $38 \%$ of the population had attained a bachelor's degree, $6 \%$ of the population had attained an associate degree, $23 \%$ of the population had some college experience, and the remaining $18 \%$ had attained a high school diploma or GED (Table 1 ). No participants had less than a high school education or equivalent. Note, of the 34 participants, only $29 \%(n=10)$ had received at least 1 dose of the HPV vaccine themselves, with just $14 \%(n=5)$ of the study population having completed the full three dose series of the HPV vaccine. Three participants chose not to answer this survey item. The remaining participants $(n=21)$ had not received any doses of the HPV vaccine.

\section{Vaccination Intentions and Perceptions}

Prior to completion of the educational session, only $35 \%(n=12)$ of participants intended to vaccinate their children according to the Advisory Committee on Immunization Practices (ACIP) recommendations by initiating the HPV vaccine series at age 1112. However, $9 \%(n=3)$ already had another child in the ACIP recommended age range or older and had begun vaccination. Another $12 \%(n=4)$ intended to vaccinate their child at a later age. Twenty-three percent $(n=8)$ of the population did not intend to vaccinate. The remaining $21 \%(n=7)$ of the population was unsure of whether they would vaccinate their children or chose not to respond. Open response item explanations for such choices were safety and efficacy concerns and lack of information. This was further confirmed during the Q\&A portion of the educational session. The pharmacist was asked about vaccine safety and side effects at all parenting group educational sessions. Following completion of the intervention, $44 \%(n=15)$ of the population intended to vaccinate at the ACIP recommended age. Another $17 \%(n=6)$ intended to vaccinate their child at an age later than 11-12. Again, $9 \%(n=3)$ had already begun vaccination. Only $12 \%(n=4)$ of the population decidedly would not vaccinate their child, while $18 \%(n=6)$ were unsure or chose not to respond. (Figure 1 and 2). Overall participants now intending to vaccinate (either at the ACIP recommended age or at a later age) increased by $14 \%$ following the intervention.

\section{Vaccine Decision-Making Process}

Of the 34 participants, $82 \%(n=28)$ indicated that they began making any vaccination decisions for their child prior to the child's birth. Of the remaining participants, $9 \%(n=3)$ indicated that they began making decisions for the child before they were one-year-old. Only 1 participant (3\%) indicated that they made vaccine decisions for the child after they were 10 years of age. The remaining 2 participants declined to answer. Participants were also asked about their influences when making vaccine decisions. In a "select all that apply" format survey item, 91\% $(n=31)$ of parents indicated that they used information from their doctor regarding vaccines. Only $6 \%(n=2)$ of parents relied on their pharmacist for vaccine information. Yet, $21 \%$ and $24 \%$ of participants used information from their family/friends and the internet, respectively. Additionally, $15 \% \quad(n=5)$ of participants used information from social media to inform their vaccine decisions (Figure 3).

\section{Awareness}

In addition, awareness of availability of the HPV vaccine at community pharmacies was assessed. Prior to the intervention, only $32 \%(n=11)$ of participants were aware that the HPV vaccination was available to be administered at their community pharmacy. Following the intervention, $100 \%(n=34)$ participants were aware that they could obtain the HPV vaccine at a community pharmacy. Of note, vaccination in West 
Virginia at the community pharmacy level continues to require a prescription for those age 11-18.

\section{Discussion}

With the preceding results, it can reasonably be concluded that educational interventions during early childhood may be effective for changing parental vaccine intentions regarding the HPV vaccine. The participating groups showed a clear change in intention, as evidenced by the increase in the number of parents/guardians intending to vaccinate at either the ACIP recommended age or a later age, and the decrease in the number of participants who were decidedly not vaccinating their child.

This study also helps to illuminate portions of the parent vaccine decision-making process. The majority of parents made vaccination decisions prior to birth or very early in the child's life. Early intervention by healthcare practitioners, such as pharmacists, is vital for contacting parents when decisions are made, rather than trying to reverse a vaccine hesitant decision. Our research also revealed that the majority of parents continue to use their physician as a primary information source. While this is promising, as physicians are an excellent source for factual vaccination information, parents also used a variety of sources not verified for medical accuracy. Healthcare providers must be aware of parents turning elsewhere for vaccine information, and be prepared to answer questions and concerns. Pharmacists can take a proactive approach by asking all patients about vaccine status and concerns at each patient encounter.

Community outreach can also increase public awareness of public health services offered at specific locations. Participants were largely unaware of the ability to receive the HPV vaccine at community pharmacies prior to the educational session, but showed universal awareness following the intervention. While the state of West Virginia did not allow pharmacies to administer the HPV vaccine to those age 11-18 prior to 2018, the service is now becoming widespread. This study shows the importance of educating the public when new public health services become available. Ideal utilization relies on an awareness of the service.

This study had several limitations which call for further research in the field. Limitations exist both in regards to study design and final study population. This study utilized a brief, single instance with a snapshot style data capture. No analysis for statistical significance could be performed due to small sample size and lack of control group. While initial results look promising, further studies must be completed to determine whether such an intervention would have impact on actual vaccination rates later in a child's life. The final study population was also predominately white and female, limiting generalizability. Non-response bias is undoubtedly present, as parenting groups specifically cited religious and political beliefs as primary reasons for non-participation. These groups represent likely vaccine hesitant parents and could significantly impact study results. Self-selection bias may also be present, as the parenting groups were formed prior to study conception by parent choice. Furthermore, additional research is needed on whether a single intervention is sufficient to affect behavior as well as achieve the maximum number of participants affected. Continued interaction between the parent/legal guardian and a public health professional or healthcare provider may provide additional impact not captured within the scope of this study.

Limitations also exist due to the legislative environment surrounding the study. Pharmacist-provided HPV vaccination was newly available at the time of intervention due to state law expansion. Given that time is an essential factor for dissemination of information, the dramatic increase in awareness of HPV vaccine availability at community pharmacies may not have been observed had the study been conducted at a later time. HPV vaccines have only been available for a short period of time in the community setting in West Virginia. As local community pharmacies adjust to the new legislation and begin roll-out of HPV vaccine advertisement campaigns, the baseline awareness is likely to be higher than what was found in this study. Follow-up research retrospectively assessing awareness in additional parenting groups would provide a comparison for current data. This would determine whether such an effect was observed due to lack of maturation of services or was a true knowledge gap for participants.

During the course of the intervention, several parents shared that their vaccine intentions were shaped by fear of vaccine side effects and other vaccine-related dangers. This was also reflected in free-form survey item responses (i.e. Other - please specify). While many organizations such as the American Public Health Association and American Pharmacists Association have worked tirelessly to increase vaccine education including the limited occurrence of vaccine side effects and the lack of an association between vaccines and autism, various negative vaccine beliefs continue to persist. Vaccine information is still garnered through multiple sources. The ever growing role of social media, the Internet and validity of information provided cannot be underscored when assessing health-decision making regarding immunizations. Social media and the Internet are plagued with personal posts, blogs and websites that are not based on scientific truths regarding immunizations. Nearly a quarter of participants that we surveyed stated that they relied on either information from family/friends, the Internet, or social media to inform their vaccine decisions. It is our responsibility as public health professionals to take note of such information availability and educate the public accordingly. 


\section{Practice Implications}

Vaccination remains of paramount importance in public health within the last decade. In a climate where vaccination decision making seems to be affected by websites, social media posts, and even Hollywood idols, public health practitioners and pharmacists are called to be at the forefront. The public requires both the availability of vaccination services and education on the benefits/risks of vaccination. Establishment of community relationships with faith-based and parental community groups will be vital for reaching vaccine-hesitant groups. Specifically, partnerships between public health officials and pharmacists and community group influencers such as parish nurses, parent group organizers, and childcare facilities must be established. This study has shown that educational outreach is a positive starting point for altering vaccination intentions. Such efforts must be continued and grown to reach Healthy People $2020^{3}$ goals. Congruently, practitioners must continue to advocate for vaccine policy change. Policy can greatly affect vaccine availability and convenience. Although policies such as the West Virginia legislation allowing vaccination of children $11-18$ year-olds with a prescription is a positive move towards vaccination goals, it is not enough. Practitioners must continue to advocate for the expansion of vaccine offerings with fewer restrictions, particularly the HPV vaccine, in all 50 states.

Creative Commons License: None

Conflicts of Interest: We declare no conflicts of interest or financial interests that the authors or members of their immediate families have in any product or service discussed in the manuscript, including grants (pending or received), employment, gifts, stock holdings or options, honoraria, consultancies, expert testimony, patents and royalties.

Treatment of Human Subjects: IRB review/approval required and obtained.

\section{References}

1. Fenner F, Henderson D, Arita I, Jezek Z, Ladnyi I. Smallpox vaccine and vaccination in the intensified smallpox eradication programme. Smallpox and its eradication. 1988:539-592.

2. Chatterjee A. The next generation of HPV vaccines: nonavalent vaccine V503 on the horizon. Expert review of vaccines. 2014;13(11):1279-1290.

3. Health UDo, Services H. The Secretary's Advisory Committee on National Health Promotion and Disease Prevention Objectives for 2020. Phase I report: Recommendations for the framework and format of Healthy People 2020. Section IV. Advisory Committee findings and recommendations. In:2008.

4. Reagan-Steiner S, Yankey D, Jeyarajah J, et al. National, Regional, State, and Selected Local Area Vaccination Coverage Among Adolescents Aged 13-17 Years--United States, 2014. MMWR Morbidity and mortality weekly report. 2015;64(29):784-792.
5. Mohammed KA, Subramaniam DS, Geneus CJ, et al. Rural-urban differences in human papillomavirus knowledge and awareness among US adults. Prev Med. 2018;109:39-43.

6. Group UCSW. United States cancer statistics: 1999-2006 incidence and mortality Web-based report. Atlanta: US Department of Health and Human Services, Centers for Disease Control and Prevention and National Cancer Institute. 2010.

7. Viens LJ. Human papillomavirus-associated cancersUnited States, 2008-2012. MMWR Morbidity and mortality weekly report. 2016;65.

8. Gilkey MB, Calo WA, Moss JL, Shah PD, Marciniak MW, Brewer NT. Provider communication and HPV vaccination: The impact of recommendation quality. Vaccine. 2016;34(9):1187-1192.

9. Islam JY, Gruber JF, Lockhart A, et al. Opportunities and challenges of adolescent and adult vaccination administration within pharmacies in the United States. Biomedical informatics insights. 2017;9:1178222617692538.

10. McLean HQ, VanWormer JJ, Chow BD, et al. Improving human papillomavirus vaccine use in an integrated health system: Impact of a provider and staff intervention. Journal of Adolescent Health. 2017;61(2):252-258.

11. Westrick SC, Hohmann LA, McFarland SJ, Teeter BS, White KK, Hastings TJ. Parental acceptance of human papillomavirus vaccinations and community pharmacies as vaccination settings: A qualitative study in Alabama. Papillomavirus Research. 2017;3:24-29.

12. Kester LM, Shedd-Steele RB, Dotson-Roberts CA, Smith J, Zimet GD. The effects of a brief educational intervention on human papillomavirus knowledge and intention to initiate HPV vaccination in 18-26 year old young adults. Gynecologic oncology. 2014;132:S9-S12.

13. Reiter PL, Stubbs B, Panozzo CA, Whitesell D, Brewer NT. $H P V$ and HPV vaccine education intervention: effects on parents, healthcare staff, and school staff. Cancer Epidemiology and Prevention Biomarkers. 2011;20(11):2354-2361

14. Winer RL, Gonzales AA, Noonan CJ, Buchwald DS. A Cluster-Randomized Trial to Evaluate a MotherDaughter Dyadic Educational Intervention for Increasing HPV Vaccination Coverage in American Indian Girls. Journal of community health. 2016;41(2):274-281.

15. Brunson EK. How parents make decisions about their children's vaccinations. Vaccine. 2013;31(46):5466-5470.

16. Glanz JM, Wagner NM, Narwaney KJ, et al. A mixed methods study of parental vaccine decision making and parent-provider trust. Academic pediatrics. 2013;13(5):481-488.

17. Loudon K, Buchanan S, Ruthven I. The everyday life information seeking behaviours of first-time mothers. Journal of documentation. 2016;72(1):24-46. 
Table 1

\begin{tabular}{|l|l|l|l|l|l|}
\hline Age & $\%(\mathbf{n})$ & Race & $\%(\mathbf{n})$ & Education Level & \% (n) \\
\hline $\mathbf{1 8 - 2 4}$ & $15 \%(n=5)$ & Caucasian & $91 \%(n=31)$ & HS Diploma/GED & $18 \%(n=6)$ \\
\hline $\mathbf{2 5 - 3 4}$ & $41 \%(n=14)$ & Asian/PI & $6 \%(n=2)$ & Some College & $23 \%(n=8)$ \\
\hline $\mathbf{3 5 - 4 4}$ & $32 \%(n=11)$ & $\begin{array}{l}\text { Native } \\
\text { American }\end{array}$ & $3 \%(n=1)$ & Associate Degree & $6 \%(n=2)$ \\
\hline $\mathbf{4 5 - 5 4}$ & $6 \%(n=2)$ & & & Bachelor's Degree & $38 \%(n=13)$ \\
\hline Over 55 & $15 \%(n=5)$ & & Graduate/Professional Degree & $15 \%(n=5)$ \\
\hline
\end{tabular}

Figure 1

Do You Intend to Vaccinate Your Child Against HPV? PreTest

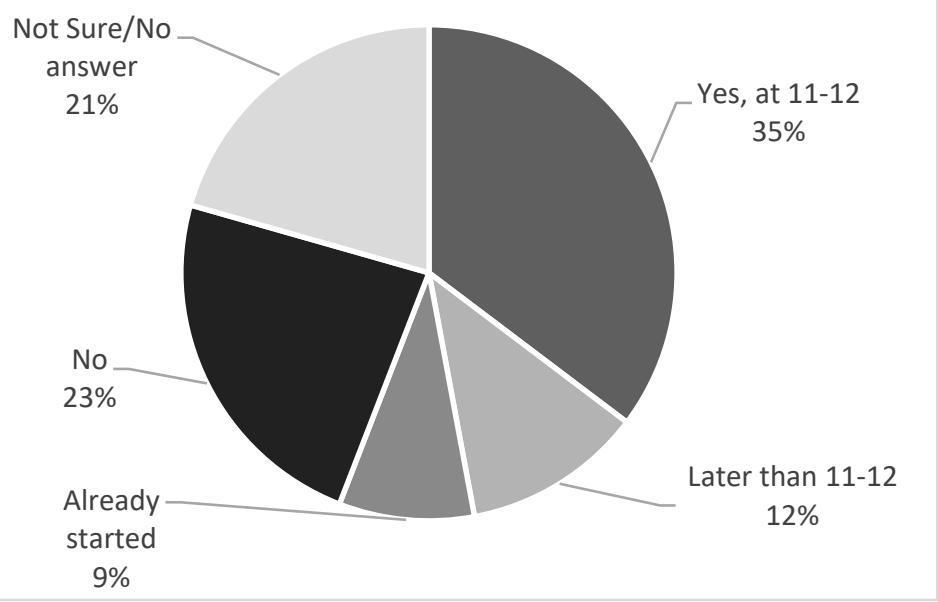

Figure 2

Do You Intend to Vaccinate Your Child Against HPV? - PostTest

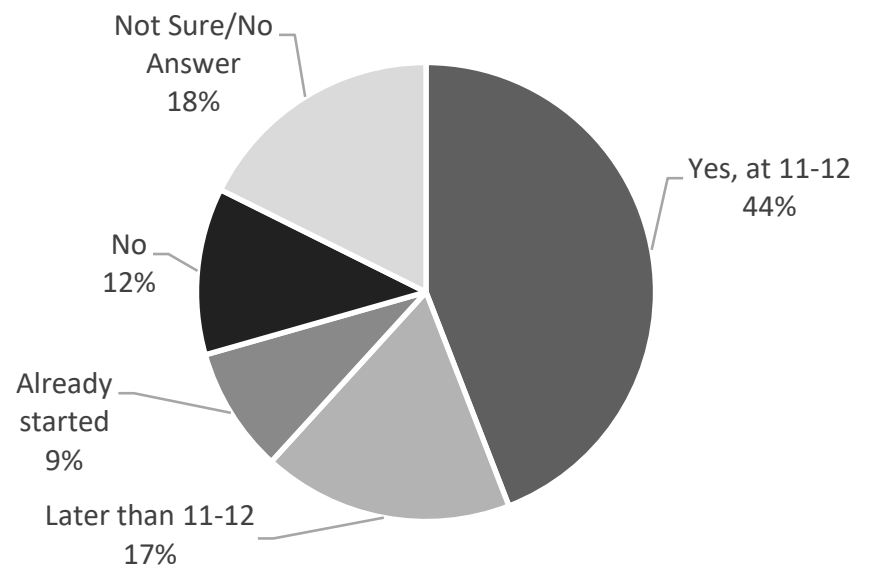

Figure 3

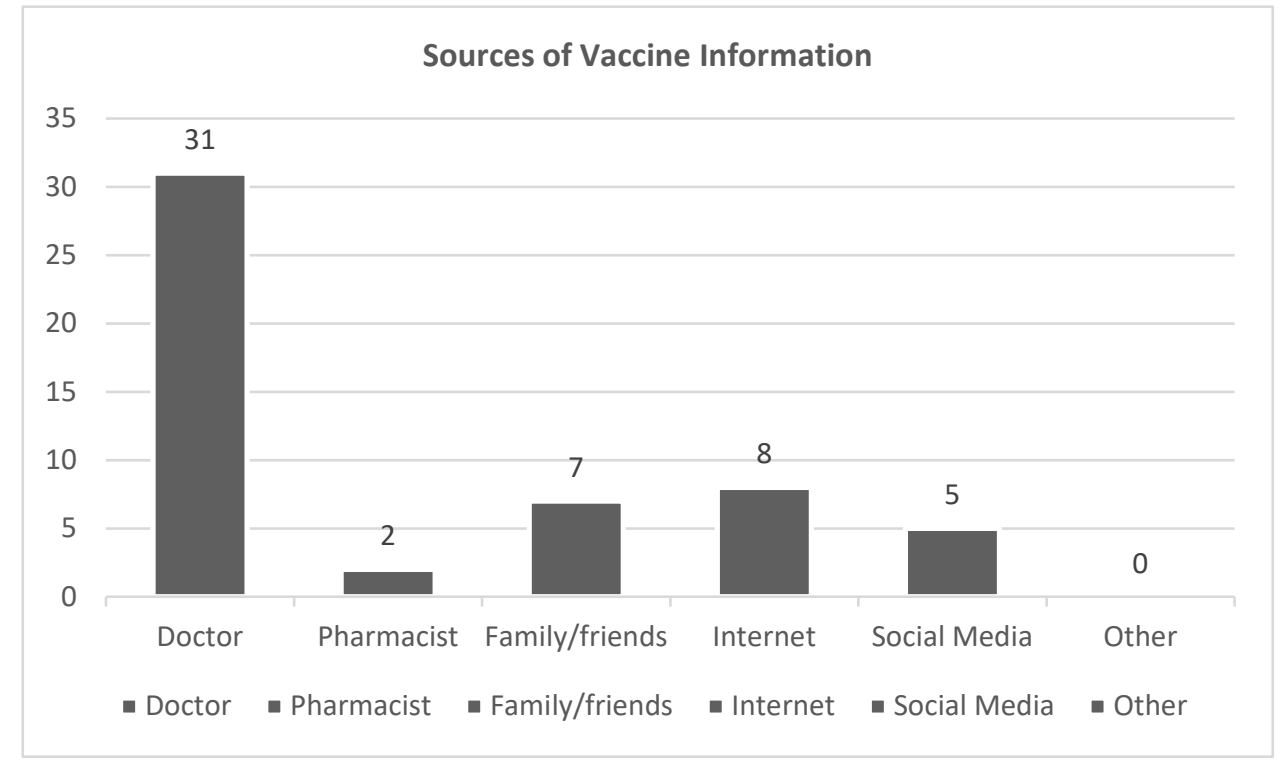

